\title{
Pressure-temperature range of reactions between liquid iron in the outer core and mantle silicates
}

\section{Xi Song and Thomas J. Ahrens}

Division of Geological and Planetary Sciences, California Institute of Technology

Abstract. The possibility of reaction between the liquid iron of the Earth's outer core and crystalline silicates $\left((\mathrm{Mg}, \mathrm{Fe}) \mathrm{SiO}_{3}\right)$ of the mantle, $\mathrm{Mg}_{0.9}, \mathrm{Fe}_{0.1} \mathrm{SiO}_{3}(\mathrm{pv})+$ $0.15 F e(\epsilon)=0.9 \mathrm{MgSiO}_{3}(p v)+0.2 \mathrm{FeO}(h p p)+0.05 \mathrm{FeSi}(\epsilon)+$ $0.05 \mathrm{SiO}_{2}(s t)$, was proposed by Knittle and Jean$\operatorname{loz}(1989,1991)$ on the basis of exploratory experiments. We calculate Gibbs free-energies for the reactants and the products of the above reaction in order to constrain the pressuretemperature range. Upon application of thermal expansion, equation of state and heat capacity data, we demonstrate that this reaction can occur at pressures as low as $30 \mathrm{GPa}$ at $3500 \mathrm{~K}$, and at temperatures as low as $900 \mathrm{~K}$ at the $130 \mathrm{GPa}$ pressure of the present core-mantle boundary. Furthermore, we propose a modified equation of reaction at the core-mantle boundary: $M g_{09} F_{2} e_{0.1} \mathrm{SiO}_{3}(p v)+0.3 \mathrm{Fe}(\epsilon)=$ $0.9 \mathrm{MgSiO}_{3}(p v)+0.3 \mathrm{FeO}(h p p)+0.1 \mathrm{FeSi}$. Our results imply that similar reactions may occur during the early accretion history of the Earth.

\section{Introduction}

The lowest $200 \mathrm{~km}$ of the mantle is designated as the $\mathrm{D}^{\prime \prime}$ layer. The complex properties of this region have received much detailed study. For example, seismologists inferred lateral heterogeneity in the $D^{\prime \prime}$ layer due to regional variation in seismic scattering at the base of the mantle(e.g. Lay, 1989). To interpret $D^{\prime \prime}$ in terms of chemical composition and physical state, Knittle and Jeanloz (1989) conducted pioneering reaction experiments. In their experiments, iron foils, embedded in a silicate perovskite matrix of a composition representative of the Earth's lower mantle, were laser heated to $>3500 \mathrm{~K}$ and the occurrence of reaction between perovskite and iron was inferred from electron microprobe scanning across the silicate-metal interface. However, the high pressures achieved in their experiments were limited to $75 \mathrm{GPa}$, substantially lower than the $133 \mathrm{GPa}$ pressure at the core-mantle boundary (CMB). To extrapolate their results to the conditions at the CMB, it is important to place their work in a theoretical framework. In this paper, we examine the pressures and temperatures required to induce several related possible reactions which may occur at the present CMB, the CMB of the earlier (hotter) Earth and at the CMB of the accreting Earth.

\section{Method}

Gibbs free energy of a single phase along an isotherm can be expressed as (e.g. Kern and Weisbrod, 1967),

$$
G(P, T)=H(0, T)-T S(0, T)+\int_{0}^{P} V(T, P) d P
$$

where

$$
\begin{aligned}
& H(0, T)=\Delta H_{298}^{0}+\int_{298}^{T} C_{p} d T \\
& S(0, T)=\Delta S_{298}^{0}+\int_{298}^{T} \frac{C_{p}}{T} d T
\end{aligned}
$$

Copyright 1994 by the American Geophysical Union.

Paper number 93GL03262

0094-8534/94/93GL-03262\$03.00
$\Delta S_{298}^{0}$ and $\Delta H_{298}^{0}$ are standard entropy of formation and enthalpy of formation from elements at $298.5 \mathrm{~K}$. $V$ and $C_{P}$ are molar volume and heat capacity at constant pressure of the phase of interest, while $T$ and $P$ are temperature and pressure of the system, respectively. Integration of $V$ is done along the isotherm at the given temperature and those of $C_{p}$ and $\frac{C p}{T}$ are along the isobar at $P=1$ bar.

An empirical form for $C_{P}$ for use at temperatures as high as $3000 \mathrm{~K}$ is (Berman and Brown, 1985),

$C_{P}(T)=\left(K_{0}+K_{1} T^{-0.5}+K_{2} T^{-2}+K_{3} T^{-3}\right)+l_{1} T+l_{2} T^{2}+l_{3} T^{3}$

and Fei et al. (1990) presents another expression for heat capacity at constant pressure,

$C_{P}(T)=3 n R\left(1+k_{1} T^{-1}+k_{2} T^{-2}+k_{3} T^{-3}\right)+(A+B T)+C_{p}^{\prime}$

where $k$, and $K$, are determined by fitting the measured intermediate temperature $(50-1000 \mathrm{~K})$ heat capacity data, $l_{B}$ are used for phases undergoing Lamda transitions, $A$ and $B$ are calculated from thermal expansion coefficient, isothermal bulk modulus, and molar volume data, and $C_{P}^{\prime}$ is due to cation disordering and is neglected as suggested by Fei et al. (1990).

For minerals in the reactions given by equations $9-10$ in the paper, we employ thermodynamic data presented by Fei et al. (1990). For other minerals discussed in this paper, thermodynamic data are from Fei and Saxena(1986). With equations 2-5, we calculate the free-energy of a single phase at atmospheric pressure as a function of temperature. In order to evaluate the integral in equation (1), we construct isothermal equations of state for several relevant minerals at high temperatures, starting from the Birch-Murnaghan equation of state. An empirical expression for the thermal expansion coefficient at ambient pressure is (Fei et al. , 1990),

$$
\alpha(T)=a_{0}+a_{1} T+a_{2} T^{-2}
$$

Thermal expansion data for many materials are available (Fei and Saxena, 1986) to evaluate the parameters in equation (6) to temperatures up to $1600 \mathrm{~K}$ (Mao et al. ,1991). We will assume that equation (6) is valid at the higher temperatures relevant to the $\mathrm{CMB}$.

The effect of pressure on the thermal expansion coefficient is (Birch, 1952),

$$
\alpha=\alpha_{0}\left(\frac{V}{V_{0}}\right)^{\delta_{T}}
$$

where $\delta_{T}$ is the second Gruneisen parameter which is assumed to be independent of temperature and pressure above Debye temperature(e.g. Anderson et al. 1991). $V_{0}$ and $\alpha_{0}$ are molar volume and thermal expansion coefficient at some reference state. Although Fei(1993) collects some $\delta_{T}$ values, we have to estimate $\delta_{T}$ for some minerals using compositionally or structurally similar ones. The values of $\delta_{T}$ we used, which are not given by $\mathrm{Fei}(1993)$, are: $\mathrm{FeO}(h p p), 4.0$; $\mathrm{FeSi}(\epsilon), 4.0 ; \mathrm{SiO}_{2}(s t), 6.0$.

Equations of state at high temperature are generated as follows: We construct a grid in the $P-T$ plane (temperature intervals of $1 \mathrm{~K}$, pressure intervals of $1 \mathrm{bar}$ ), and first consider the $298 \mathrm{~K}$ isotherm centered at $P=1$ bar. Molar volume of a mineral along this isotherm is calculated using a third order Birch-Murnaghan equation of state(Birch, 
1952). The molar volumes at $P=1$ bar and a higher temperature $T$, are calculated using equation (6) together with the definition of the thermal expansion coefficient,

$$
V=V_{0} \exp \left(\int_{T_{0}}^{T} \alpha(T) d T\right)
$$

We then calculate the thermal expansion coefficient at higher temperatures and pressures with equation (7) along an isotherm and calculate the molar volume at higher temperatures and pressures with equation (8) along an isobar. Upon the completion of these extrapolations, the equation of state at a given high temperature $T$ is obtained in the form $V_{\mathrm{i}}=V_{i}\left(P_{1}, T\right)$.

To examine the validity of this method, we compared our calculations with experimental high temperature and high pressure equations of state for perovskite $(p v)$. On the basis of high temperature( up to $1150 \mathrm{~K}$ ) and high pressure(up to $30 \mathrm{GPa})$ experiments on $(\mathrm{Mg}, \mathrm{Fe}) \mathrm{SiO}_{3}$ perovskite, Mao et al. (1991) gave two fits for the $V-T-P$ equation of state, specified as fit A and fit B. Fei et al. (1992) conducted similar study on $\left(M g_{0.6}, F e_{0.4}\right) O$ magnesiowustite and gave the $V-T-P$ equation of state as their equation (8). In figure 1 , we compare isotherms at $1100 \mathrm{~K}, 1500 \mathrm{~K}$ and $2000 \mathrm{~K}$ calculated from our extrapolations with the equations of state from Mao et al. (1991) and Fei et al. (1992). There is excellent agreement between the two methods.

Using our extrapolation method and the heat capacity formulae (4) and (5), we study two known reactions of geophysical interest. Firstly, we study

$$
\mathrm{Mg}_{2} \mathrm{SiO}_{4}(\beta)=\mathrm{Mg}_{2} \mathrm{SiO}_{4}(\gamma)
$$

by calculating the Gibbs free energies of $\beta$-spinel and $\gamma$ spinel along the $1600 \mathrm{~K}$ isotherm. At lower pressure, $\beta$ spinel has a Gibbs free energy that is lower than that of $\gamma$ spinel. With the increasing pressure, the difference between the Gibbs free energies of these two phases decreases. At pressure $P=17.5 \mathrm{GPa}$, these two phases coexist and are in equilibrium. This result agrees with Akaogi et al. 's experiments (1989) which gave the transition pressure at $1600 \mathrm{~K}$ to be 16-18 GPa. Secondly, we study

$$
\mathrm{Mg}_{2} \mathrm{SiO}_{4}(\gamma)=\mathrm{MgO}(\mathrm{mw})+\mathrm{MgSiO}_{3}(p v)
$$

The calculation shows the transition pressure for the postspinel at $1600 \mathrm{~K}$ is $23.7 \mathrm{GPa}$, which is in agreement with experimentally determined transition pressure $23.1 \mathrm{GPa}$ (Ito et al. ,1989).

\section{Prediction on the Possible Reactions at the Core-Mantle Boundary}

Knittle and Jeanloz(1991) proposed that the following reaction occurred in their experiments and it may be representative of reactions between lower mantle silicates and liquid iron at the $\mathrm{CMB}$,

$$
\begin{gathered}
\left(\mathrm{Mg}_{0.9}, \mathrm{Fe}_{0.1}\right) \mathrm{SiO}_{3}(p v)+0.15 \mathrm{Fe}(\epsilon)=0.9 \mathrm{MgSiO}_{3}(p v) \\
+0.2 \mathrm{FeO}(h p p)+0.05 \mathrm{FeSi}(\epsilon)+0.05 \mathrm{SiO}_{2}(s t)
\end{gathered}
$$

To calculate the pressure and temperature conditions required for this reaction, we assumed that equation (4) and (5) are valid even at high temperatures of the CMB. Moreover, we make a few assumptions for iron, the low and high pressure phases of $\mathrm{FeO}$ and $\mathrm{FeSi}$.

\section{$\underline{\epsilon-I r o n}$}

According to Brown and McQueen (1982), The high pressure phase of iron stable at the pressures of the CMB may be $\epsilon$-iron. Huang and Bassett(1987) studied the equations of state of $\alpha$ - and $\epsilon$-iron and gave the elastic parameters. Thermal expansion data for $\epsilon$-iron are not available, but our tests of using $\alpha$-iron data(Fei et al. , 1986) and $\gamma$-iron data(Boehler et al. , 1990) as an approximation show that the free-energy of reaction (11) is relatively insensitive to the thermal expansion model. Thermal expansion data for
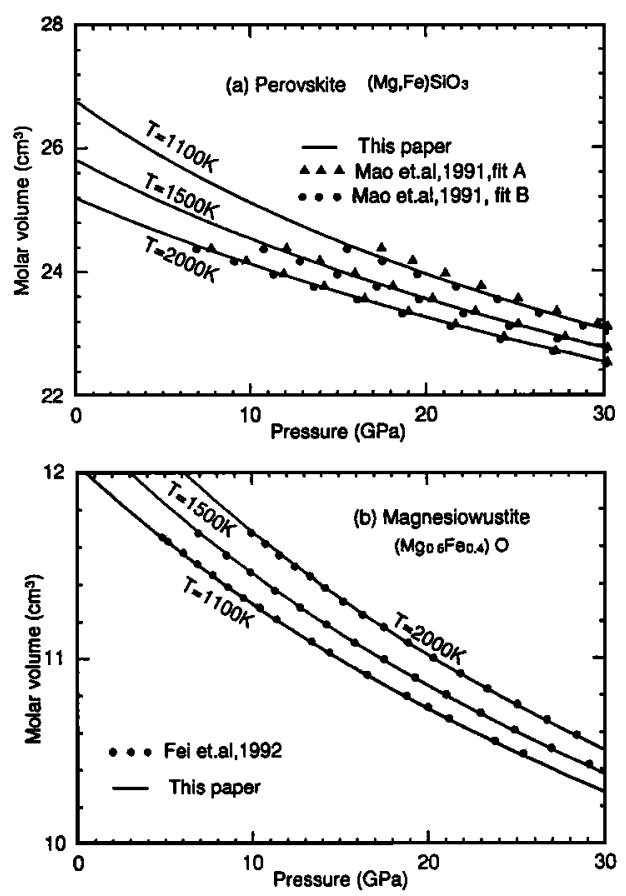

Fig. 1. Isothermal equations of state at $1100 \mathrm{~K}, 1500 \mathrm{~K}$ and $2000 \mathrm{~K}$, for (a) perovskite $\left((\mathrm{Mg}, \mathrm{Fe}) \mathrm{SiO}_{3}\right)$ and (b) magnesiowustite $\left(\left(M g_{0.6} F e_{0.4}\right) O\right.$.

$\alpha$-iron are used in this paper for the liquid phase of iron in the outer core. Specific heats for $\epsilon$-iron are not available either but that for $\alpha$-iron is given by Fei et al. (1986). Moreover, Andrews (1973) calculated the Gibbs potential difference between $\epsilon$-iron and $\alpha$-iron at atmospheric pressure and various temperature up to $800 \mathrm{~K}$. Their difference, decrease linearly with increasing temperature, do not exceed $5 \mathrm{KJ} / \mathrm{mol}$ and can be fit with

$$
G_{\epsilon}-G_{\alpha}=1232.067-0.5043 T
$$

We adopt these values to calculate the Gibbs free energy at 1 atm pressure for $\epsilon$-iron from that of $\alpha$-iron.

\section{Iron-Silicon Alloy}

Thermal expansion data of iron-silicon alloys are not available and we adopted thermal expansion data of $\alpha$-iron. Chart (1970) studied the thermodynamic properties of the system $\mathrm{Fe}-\mathrm{Si}$ and presented thermodynamic data for the $\epsilon$ phase of $\mathrm{FeSi}(\epsilon)$. Heat capacities at atmospheric pressure and temperature lower than $1683 \mathrm{~K}$, the melting point, as presented in Chart (1970), are fitted by formula (4) with the coefficients: $k_{0}=113.453, k_{1}=-1.963 \times 10^{3}$, $k_{2}=9.6 \times 10^{6}, k_{3}=-1.599 \times 10^{9}$. In this paper, heat capacities of this phase at atmospheric pressure and temperatures higher than $1683 \mathrm{~K}$ are assumed to be constant and equal to $87.78 \mathrm{~J} / \mathrm{mol} . \mathrm{K}$, as indicated by Chart's data. Using high temperature heat capacity data that are extrapolated from those at lower temperatures will lower the transition pressures and temperatures of reaction (11), but will not alter our conclusions.

\section{High pressure phase of $\mathrm{FeO}$}

At the CMB, the $\mathrm{FeO}$ formed via equation (11) presumably occurs in a high pressure phase (Jeanloz and Ahrens, 1980). Although a bulk modulus and molar volume of this high pressure phase of $\mathrm{FeO}$ is reported by Jeanloz and Ahrens (1980), the thermal expansion data for this phase is still not available, and we resort to using thermal expansion coefficient of the low pressure phase of $\mathrm{FeO}$ for the high pressure phase. Heat capacities calculated from equation (4) with the coefficients for the low pressure phase (Fei and Saxena, 1986), do not appear appropriate for the high pressure phase of $\mathrm{FeO}$. Instead, we adopt the expression 


$$
C_{p}=3 n R+\alpha^{2} V T K_{T}
$$

derived from the Dulong Petit value for this high pressure phase of $\mathrm{FeO}$. Here $n$ is the number of atoms per formula unit, $\alpha$ is thermal expansion coefficient expressed in equation (6), $V$ is the molar volume in equation (8), and $K_{T}$ is the bulk modulus. Again we assume that the product $\alpha K$ is constant and independent of temperature(Birch, 1952).

On the basis of these assumptions, and neglecting the possible effect of solid solution, we calculated the total Gibbs free-energies at atmospheric pressure and various temperatures for the reactants and the products of the proposed reaction(11). In figure 2 , the differences of the total Gibbs free energies between the reactants and products are plotted along $2000 \mathrm{~K}, 3000$ and $3500 \mathrm{~K}$ isotherms. The same procedure was applied to other isotherms and we derived a $\mathrm{P}-\mathrm{T}$ diagram for reaction (11) in figure 3 . The breaks in slope of the curves in this figure are due to the use of the heat capacity data of $\mathrm{FeSi}$ at atmospheric pressure and temperatures higher than $1683 \mathrm{~K}$.

\section{Discussion and Conclusions}

Figures 2-3 have a large formal uncertainty. As shown in figure 2 , the difference between the energies of the products and the reactants does not exceed $15 \mathrm{KJ}$ per mole atoms, but the uncertainty of $\Delta H$, as indicated in some of the references(e.g. Fei et al. ,1990), can be as great as $1 \mathrm{KJ}$ per mole. This in turn may result in a large uncertainty of transition pressure on the order of $100 \mathrm{GPa}$. Besides, we do not have a clear idea about how much uncertainty is involved by extrapolating isotherms from lower temperaturepressure condition to extremly high temperatures and pressures. A possible mitigating circumstance would be if the error in the measurements of $\Delta H$ for individual reactants and products cancel out for the reaction, as we tried to use a self-consistent data set for each reaction. Nevertheless, within the uncertainty, the results of figure 3 are in excellent agreement with the experimental results of Knittle and Jeanloz(1991).

Of importance is that, as pressure increases along the isotherm, the difference in free energies between the reactants and the products increases, and the products become more and more stable relative to the reactants, as shown in figure 2. Also it should be noted that, as temperature increases, the transition pressure decreases. Therefore, to some extent, this result extends the conclusion of Knittle and Jeanloz(1991) to high pressure-temperature conditions relevant to the CMB.

Moreover, our results suggest that reaction between iron and silicates could even occur at lower temperatures and lower pressures than those at the CMB, provided perovskite, the high pressure phase of $\mathrm{FeO}$ and stishovite can exist stably. These conditions could be achieved in the center of the present Mars, and could have been achieved within Venus and Earth much earlier during their accretion history. Thus, our results could explain the compositional difference in iron content between the Martian mantle and the Earth's mantle.

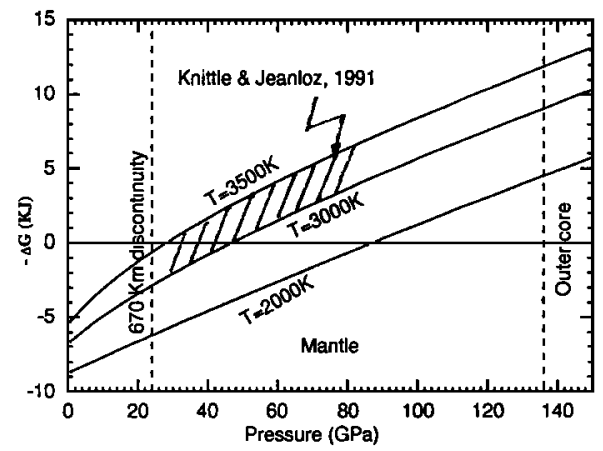

Fig. 2. Gibbs free energy of reaction for Knittle and Jeanloz's(1991) proposed equilibrium(equation (11)), along three isotherms, in $K J$. Uncertainties are discussed in the text.

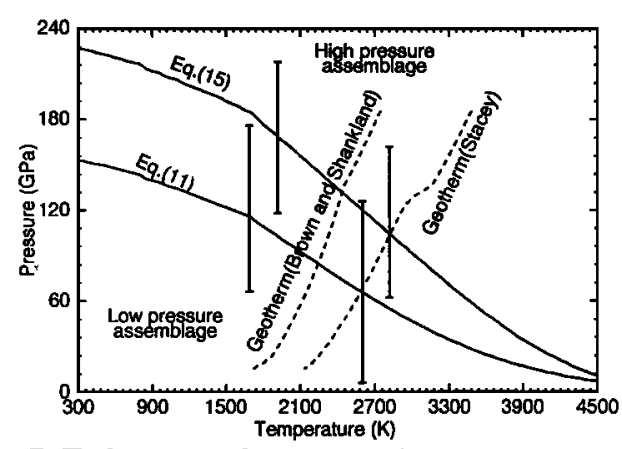

Fig. 3. P-T diagram showing stability field for reactant and product phase assemblages, in equation (11) and (15); Error bars show estimated uncertainties. Also shown are two mantle geotherms derived from the thermal models of Stacey (1977) and Brown and Shankland(1981), with pressures given by PREM (Dzeiwonski et al. , 1981).

The same approach can be applied to another reaction

$$
\mathrm{SiO}_{2}(s t)+3 \mathrm{Fe}(\epsilon)=2 \mathrm{FeO}(h p p)+\mathrm{FeSi}
$$

We find there is also a possibility that $\mathrm{SiO}_{2}$ reacts with $\epsilon$-iron at conditions at the CMB. This is in accord with Knittle and Jeanloz's observation that little stishovite appeared as a product. In fact, our calculations suggest a modified equation of reaction in the form

$$
\begin{aligned}
& \mathrm{Mg}_{0.9}, \mathrm{Fe}_{0.1} \mathrm{SiO}_{3}(p v)+0.3 \mathrm{Fe}(\epsilon) \\
= & 0.9 \mathrm{MgSiO}(p v)+0.3 \mathrm{Fe} \mathrm{O}(\mathrm{hpp})+0.1 \mathrm{FeSi}
\end{aligned}
$$

The $P-T$ diagram for this reaction is also shown in figure 3 . In terms of Gibbs free energy, reaction (15) occurs at higher pressure than the reaction originally proposed by Knittle and Jeanloz(1991).

The reactions discussed above are essentially concerned with the partitioning of iron in $\mathrm{FeSiO}_{3}$ and iron alloys. What these calculations tell us is that, at the CMB, iron will react with lower mantle silicates to form $\mathrm{Fe}$-Si alloy, in order to minimize the Gibbs free-energy. Since the composition of the lower-most mantle is not yet well understood and all these reactions may occur at lower pressures during Earth accretion, our discussion about the possible reactions at the CMB will not be complete if we do not also consider other reactions involving different phases of mantle silicates(e.g. $\mathrm{MgSiO}_{3}$ and $\left.\mathrm{Mg}_{2} \mathrm{SiO}_{4}\right)$. Therefore, with the same approach, we studied the equation

$\mathrm{MgSiO}_{3}(p v)+3 \mathrm{Fe}(\epsilon)=\mathrm{MgO}(m w)+\mathrm{FeSi}+2 \mathrm{Fe} \mathrm{O}(h p p)$

It turns out that this reaction (16) has a very positive freeenergy of formation and is therefore unlikely to occur in terrestrial planets even at very high temperatures $(>4500 \mathrm{~K}$ ) and pressures $(>300 \mathrm{GPa})$. This suggests that the reaction between $\mathrm{Mg}_{0.9} \mathrm{Fe}_{0.1} \mathrm{SiO}_{3}$ and iron will slow down as it proceeds, due to the increasing dominance of $\mathrm{MgSiO}_{3}$

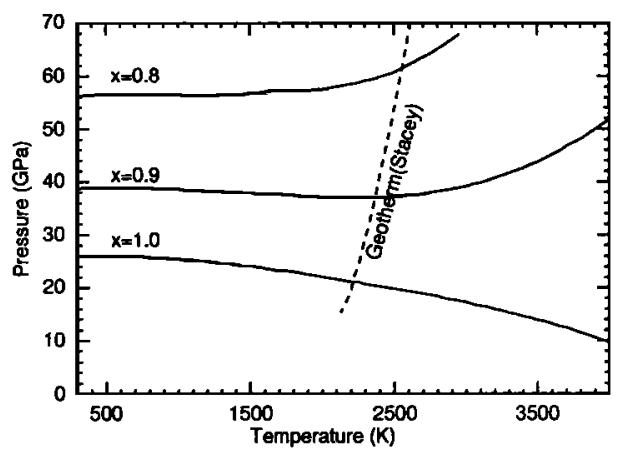

Fig. 4. P-T diagram similar to figure 3 but for the reaction between $\mathrm{Fe}(\epsilon)$ and $\mathrm{Mg}_{2} \mathrm{SiO}_{4}(\gamma) \cdot(3-3 \mathrm{x})$ is the coefficient of $\mathrm{Fe}$ in equation (17) for this reaction. 
in the composition of the lower-most mantle, unless a dynamic mechanism exists sweeping away the reaction zone and exposing fresh mantle material to the core.

Other equations examined in this study include

$$
\begin{array}{r}
\mathrm{Mg}_{2} \mathrm{SiO}_{4}(\gamma)+(3-3 x) \mathrm{Fe}(\epsilon)=x \mathrm{MgSiO}_{3}(p v)+ \\
(2-x) \mathrm{MgO}+(1-x) \mathrm{FeSi}+(2-2 x) \mathrm{FeO}
\end{array}
$$

and similar reactions with various phases of olivine and iron. These reactions, if possible, can only occur under certain conditions with much lower temperatures and pressures than those of the present CMB, due to the transformation of the $\mathrm{Mg}_{2} \mathrm{SiO}_{4}$ polymorphs; But these temperaturepressure conditions may still be achieved within the earlier, smaller proto-Earth or Venus. However, our calculations show that, among these reactions, those with $x=1.0$, which denote transforms of the $\mathrm{Mg}_{2} \mathrm{SiO}_{4}$ polymorphs, may occur at lower temperatures and lower pressures than those with $x<1.0$ and thus involve iron (see figure 4), indicating that the available data does not suggest the reaction between iron and pure iron-free $\mathrm{Mg}_{2} \mathrm{SiO}_{4}$.

In conclusion, we developed a series of high temperature equations of state to calculate Gibbs free-energies for several minerals of interest. Our calculations of the temperature-pressure range of the reaction between iron and $\mathrm{Mg} g_{0.9} \mathrm{Fe}_{0.1} \mathrm{SiO}_{3}$ are consistent with the experimental results of Knittle and Jeanloz $(1989,1991)$. If $\mathrm{SiO}_{2}(s t)$ is not involved in reactions at the CMB, we propose an occurrence of reaction between $\mathrm{Fe}$ and $\mathrm{M} g_{0.9} \mathrm{Fe}_{0.1} \mathrm{SiO}_{3}$, producing $\mathrm{MgSiO}_{3}, \mathrm{FeO}$ and $\mathrm{FeSi}$. These two reactions have the effect of making the $D^{\prime \prime}$ layer and the outer core rich in $\mathrm{FeSi}$ and $\mathrm{FeO}$. Our calculations imply that reactions (11) and (15) may occur under the lower temperature-pressure conditions at the proto-Earth's CMB during its accretion history. Moreover, the result that core iron does not tend to react with pure iron-free polymorphs of $\mathrm{Mg}_{2} \mathrm{SiO}_{4}$ and $\mathrm{MgSiO}_{3}$ suggests that iron is drained via reactions (11) or (15) from the lowermost mantle into the core, which would have been a mechanism for core formation and core-mantle segregation.

Acknowledgements We thank Don L. Anderson, Joel Ita and John Beckett for their comments and suggestions. Research supported by National Science Foundation. Division of Geological and Planetary Sciences, California Institute of Technology. Contribution 5324.

\section{References}

Akaogi, M., Eiji Ito, and Alexandra Navrotsky, Olivinemodified spinel-spinel transitions in the system $\mathrm{Mg}_{2} \mathrm{SiO}_{4}-\mathrm{Fe}_{2} \mathrm{SiO}_{4}$ : calorimetric measurements, thermochemical calculation, and geophysical application, J. Geophys. Res., 94, 15671-15685, 1989.

Anderson, O. L., Isaak D. L., and Oda, H., Thermoelastic parameters for six minerals at high temperature, $J$. Geophys. Res., 96, 18037-18046, 1991.

Andrews, D. J., Equation of state of the alpha and epsilon phase of iron, J. Phys. Chem. Solids, 34, 825-840, 1973.

Berman, R. G. and Brown, T. H., Heat capacity of minerals in the system $\mathrm{Na}_{2} \mathrm{O}-\mathrm{K}_{2} \mathrm{O}-\mathrm{CaO}-\mathrm{MgO}-\mathrm{FeO}-$ $\mathrm{Fe}_{2} \mathrm{O}_{3}-\mathrm{Al}_{2} \mathrm{O}_{3}-\mathrm{SiO}_{2}-\mathrm{TiO}_{2}-\mathrm{H}_{2} \mathrm{O}-\mathrm{CO}_{2}$ : representation, estimation and high temperature extrapolation. Contrib Mineral Petrol, 89, 168-183, 1985.

Birch, R., Elasticity and constitution of the Earth's Interior. J. Geophys. Res., 57, 227-286, 1952.

Boehler, R., N. Von Bargen, and A. Chopelas, Melting, thermal expansion and phase transitions of iron at high pressure, J. Geophys. Res., 95, 21731-21736, 1990.

Brown J. M. and R. G. McQueen, The equation of state for iron and the Earth's core, in High-Pressure Research in Geophysics, Edited by S. Akimoto and M. H. Mangh- nani, Center for Academic Publications, Tokyo, 12, 611-623, 1982.

Brown J. M. and T. J. Shankland, Thermodynamic parameters in the Earth as determined from seismic profiles, Geophy. J. R. Astr. Soc., 66, 579-596, 1981.

Chart, T. G., A critical assessment of the thermodynamic properties of the system iron-silicon, High Temperatures-High Pressures, 2, 461-470, 1970.

Dziewonski, A. M. and D. L. Anderson, Preliminary reference Earth model, Phys. Earth Planet. Inter., 25, 297-356, 1981.

Fei, Y., Thermal expansion, 1993, in press. Contributed to Handbook of Physical Constants, edited by Thomas J. Ahrens, published by American Geophysical Union, 1993 , in press.

Fei, Y., Ho-Kwang Mao, Jinfu Shu, and Jingzu Hu, P-V-T equation of state of magnesiowustite $\left(M g_{0.6} F e_{0.4}\right) O$, Phys. Chem. Minerals, 18, 416-422, 1992.

Fei, Y., and Surendra K. Saxena, A thermochemical data base for phase equilibria in the system $\mathrm{Fe}-\mathrm{Mg}-\mathrm{Si}-\mathrm{O}$ at high pressure and temperature, Phys. Chem. Minerals 13, 311-324, 1986.

Fei, Y., and Surendra K. Saxena, Internally consistent thermodynamic data and equilibrium phase relations for compounds in the system $\mathrm{MgO}-\mathrm{SiO}_{2}$ at high pressure and high temperature, J. Geophys. Res. 95, 6915-6928, 1990.

Huang E., W. A. Bassett, and P. Tao, Pressuretemperature-volume relation for hexagonal close packed iron determined by synchrotron radiation, $J$. Geophys. Res., 92, 8129-8235, 1987.

Ito, E., and E. Takahashi, Postspinel transformation in the system $\mathrm{Mg}_{2} \mathrm{SiO}_{4}-\mathrm{Fe}_{2} \mathrm{SiO}_{4}$ and some geophysical implications, J. Geophys. Res., 94, 10637-10646, 1989.

Jeanloz, R. and T. J. Ahrens, Equations of state of $\mathrm{FeO}$ and $\mathrm{CaO}, G$. J. R. Astr. Soc., 62, 505-528, 1980.

Jeanloz, R. and F. M. Richter, Convection, composition, and the thermal state of the lower mantle, J. Geophys. Res., 84, 5497-5504, 1979.

Kern, R. and A. Weisbrod, Thermodynamics for geologists. Chapter 7. Published by Masson and Cie, 1964.

Knittle, E. And R. Jeanloz, Earth's core-mantle boundary: results of experiments at high pressures and temperatures, Science, 251, 1438-1443, 1991.

Knittle, E., and R. Jeanloz, Simulating the core-mantle boundary: An experimental study of high-pressure reactions between silicates and liquid iron. Geophys. Res. Lett., sl 16, 609-612, 1989.

Lay, T., Structure of the core-mantle transition zone: a chemical and thermal boundary layer, EOS, 70, 49-59, 1989.

Mao, H. K., R. J. Hemley, Y. Fei, J. F. Shu, L. C. Chen, A. P. Jephcoat, Y. Wu and W. A. Bassett, Effect of pressure, temperature, and composition on lattice parameters and density of $(\mathrm{Fe}, \mathrm{Mg}) \mathrm{SiO}_{3}$-perovskites to $30 \mathrm{GPa}, J$. Geophys. Res., 96, 8069-8079, 1991.

Stacey D. F., A thermal model of the Earth, Phys. Earth Planet. Inter., 15, 341-348, 1977.

T.J. Ahrens and Xi Song, Division of Geological and Planetary Sciences, California Institute of Technology, Pasadena, CA 91125.

(Received September 10, 1993; accepted November 11, 1993.) 